\title{
A Frozen Spin Target for Neutron Diffraction Measurements of Proteins Crystals
}

\author{
Josh Pierce* \\ Oak Ridge National Laboratory \\ E-mail: piercejjeornl.gov
}

\begin{abstract}
Neutron scattering from protein crystals has been used successfully to determine protein structure. This technique achieves its best results when used with large crystal samples, or with samples that are amenable to techniques such as deuteration. In the case of small or delicate protein crystals, sample polarization has been identified as a method to greatly increase the signal to noise ratio in neutron diffraction studies of protein structure. The strong polarization dependence of the neutron scattering cross section of hydrogen would allow us to use Dynamic Nuclear Polarization to drastically reduce the time it takes to get reliable data from protein crystal diffraction, and enable measurements of protein structures that are currently impossible. We present a new frozen spin target being built at Oak Ridge to polarize single protein crystals on the IMAGINE beamline at the High Flux Isotope Reactor. This target will be optimized for very small samples, and built largely from "off-the-shelf" commercial items.
\end{abstract}

XVIth International Workshop in Polarized Sources, Targets, and Polarimetry, PSTP2015,

14-18 September 2015,

Bochum, Germany

\footnotetext{
${ }^{*}$ Speaker.
} 


\section{Introduction}

Neutron protein crystallography is a useful tool to determine the structure of proteins, especially in complement with more standard X-ray crystallography. Specifically, the high scattering cross section of neutrons on some light nuclei gives neutrons access to structural information that is hard to get using $\mathrm{X}$-rays, which are more sensitive to the higher $\mathrm{Z}$ atoms in the molecule. The limitations of low neutron flux compared to that of an X-ray source have limited the results that can be extracted from neutron protein crystallography. In order to get useful data from a neutron experiment, the experimenter is forced to grow very large crystals, perform extensive isotopic substitution, run very long experiments, or most likely, some combination of all of these. In many cases, none of these approaches are possible. The spin dependence of the scattering cross section for thermal neutrons was long ago recognized as a potential way to overcome these challenges, and greatly increase the usability of neutron scattering for determining protein structures.

\section{Spin Dependent Neutron Scattering}

The spin dependent scattering cross section of a thermal neutron from identical (non-spin zero) nuclei in a crystal of $N$ unit cells can be written as in equation 2.1 [1].

$$
\frac{d \sigma}{d \Omega}=N\left(\frac{d \sigma}{d \Omega}\right)_{i n c}+N\left(\frac{d \sigma}{d \Omega}\right)_{c o h} \sum_{i} \exp \left(\mathbf{Q} \cdot \mathbf{r}_{\mathbf{i}}\right)
$$

It is important to note that for the case of diffraction studies where the positions of the hydrogen atoms are the data of interest, the signal size is determined by the coherent scattering from hydrogen, $\left(\frac{d \sigma}{d \Omega}\right)_{c o h}$, whereas the dominant background comes from the incoherent scattering from hydrogen, $\left(\frac{d \sigma}{d \Omega}\right)_{i n c}$. The coherent (2.2) and incoherent (2.3) cross sections can be written in terms of the neutron polarization $P(n)$, the hydrogen polarization $P(H)$, the nuclear scattering amplitude $b$, and the spin dependent scattering amplitude $B$. These terms have been experimentally determined. For hydrogen $b=-0.375 \times 10^{-12} \mathrm{~cm}$ and $B=2.91 \times 10^{-12} \mathrm{~cm}$.

$$
\begin{gathered}
\left(\frac{d \sigma}{d \Omega}\right)_{c o h}=b^{2}+2 b B I P(n) P(H)+B^{2} I^{2} P^{2}(H) \\
\left(\frac{d \sigma}{d \Omega}\right)_{i n c}=B^{2}\left[I(I+1)-P(n) P(H) I-P^{2}(H) I^{2}\right]
\end{gathered}
$$

With the use of modern super mirror polarizers, neutron polarizations in excess of $95 \%$ can be obtained. In the limiting case of $P(n)=1$, we can plot the coherent and incoherent cross sections, as in figure 1. Since the incoherent scattering from the hydrogen is often the dominant background for crystals soaked in normal water, it is easy to see how a large hydrogen polarization could lead to a substantial improvement in signal to noise. There are further gains to be made, since the ability to resolve the locations of the hydrogen nuclei is determined by the coherent scattering, the cross section for which is increases for high proton polarization. This has been demonstrated in neutron scattering studies of proteins in solution [2], but has not yet been applied to the much more information-rich system of protein crystals. 


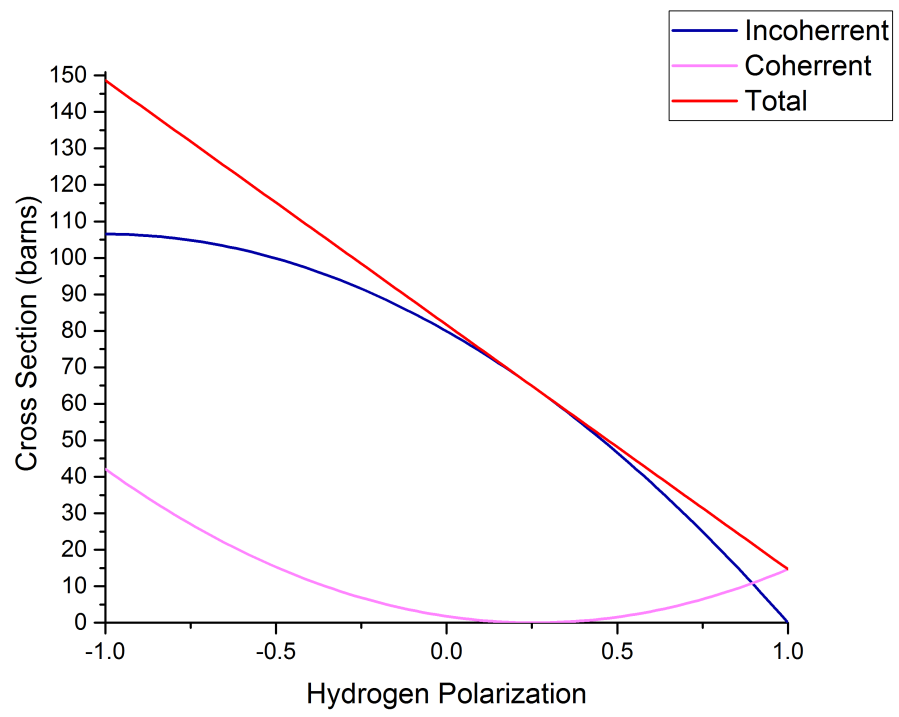

Figure 1: The integrated coherent and incoherent cross sections for polarized neutron scattering off of a polarized hydrogen nucleus, as a function of proton polarization.

\section{Target}

\subsection{Concept}

The goal is to construct a device which allows for very high hydrogen polarization of a sample composed of a single protein crystal. These crystals are very small, usually less than $1 \mathrm{~mm}$ on a side. In order to fit into existing infrastructure and detectors, a frozen spin target [3] has been identified as a means to make a device that takes up as little space on the beamline as possible. In order to conduct useful studies, it is essential that the crystal be rotated through $\approx 180^{\circ}$. The system would also require that the crystal be changed every few days, in order to measure a new protein. The target will be used on the CG-4D beamline, at the High Flux Isotope Reactor (HFIR) at Oak Ridge National Laboratory. The final design will interface with the IMAGINE instrument [4], which is a quasi-laue diffractometer based on a neutron image plate. The frozen spin target will be vertical with long narrow tail section, so that it can fit through the existing hole in the diffractometer which is $\approx 100 \mathrm{~mm}$. Initial tests may be done on the CG-4D beamline without the detector. In this case a image plate would be used to measure the diffraction patterns and read off-line.

\subsection{Requirements}

Due to the unique, very small nature of the target material the requirements for this target are different than those for a traditional frozen spin targets. For example, the small target will take very little microwave power to polarize, as long as the microwaves are well coupled to the sample. There are, however, some extra requirements not typically present for a frozen spin target. The nature of the neutron beam is such that the detector is extremely sensitive to structured backgrounds, such as those that can be caused by the micro-structure in aluminum. Also, the neutron cross sections are 


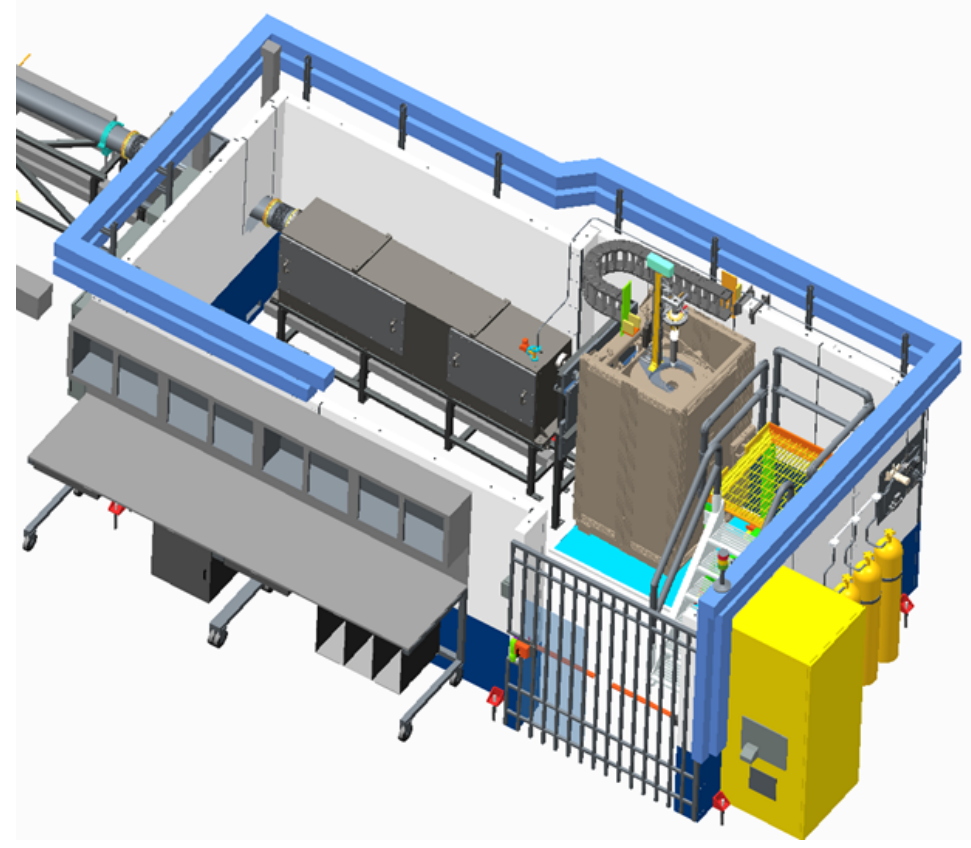

Figure 2: A model of the IMAGINE instrument, located on beamline CG-4D at HFIR

very different than those for electrons or gammas, so some of the materials used for thin windows or holding coils that work fine for these particles would be incompatible with neutrons. Finally, the sample has to be able to rotate and be changed easily.

\subsection{Design}

Theses requirements have been addressed by using a commercial, cryogen free, dilution refrigerator, with a custom sample space and tail section, and a cryogen free, warm bore $5 \mathrm{~T}$ solenoid. The use of the commercial refrigerator has a number of advantages, most obviously a shortened production time and well known performance. These refrigerators cannot generally be used for targets, as their cooling power is orders of magnitude lower than what would be required for a nuclear physics target of any reasonable size, but for the case of these very small targets, it is sufficient. The tails will be custom made, as will the sample space, including the holding coil. The coil will be hand wound, constructed of thin super conductor thermally attached to the still radiation shield, similar to the one used in reference [5]. The goal for the holding field is $0.5 \mathrm{~T}$. The main difference is that the low energy neutrons would be absorbed by the Formvar insulation of the wire, so a gap for the scattered particles must remain unobstructed. This will greatly reduce the uniformity of the holding field, but this should not be a concern, due to the extremely small sample size. A cross sectional view of the sample space is shown in figure 3, as is a model of the target as it will be mounted on the CG-4D beamline.

The microwave system does not require a high power source, and will be powered by a relatively small $140 \mathrm{GHz}$ diode source. The NMR system will be built a round a series tuned capacitor circuit modeled after the one used in the Liverpool Q-meter [6], with the detector portions of the Q-meter being replaced with network analyzer. These systems have been chosen to lower cost and 
speed up development, but in both cases, the traditional solution is still available, that being and EIO tube for the microwaves and a Liverpool Q-meter for NMR. Additionally, a $70 \mathrm{GHz}$ microwave system is available for operation at $2.5 \mathrm{~T}$.
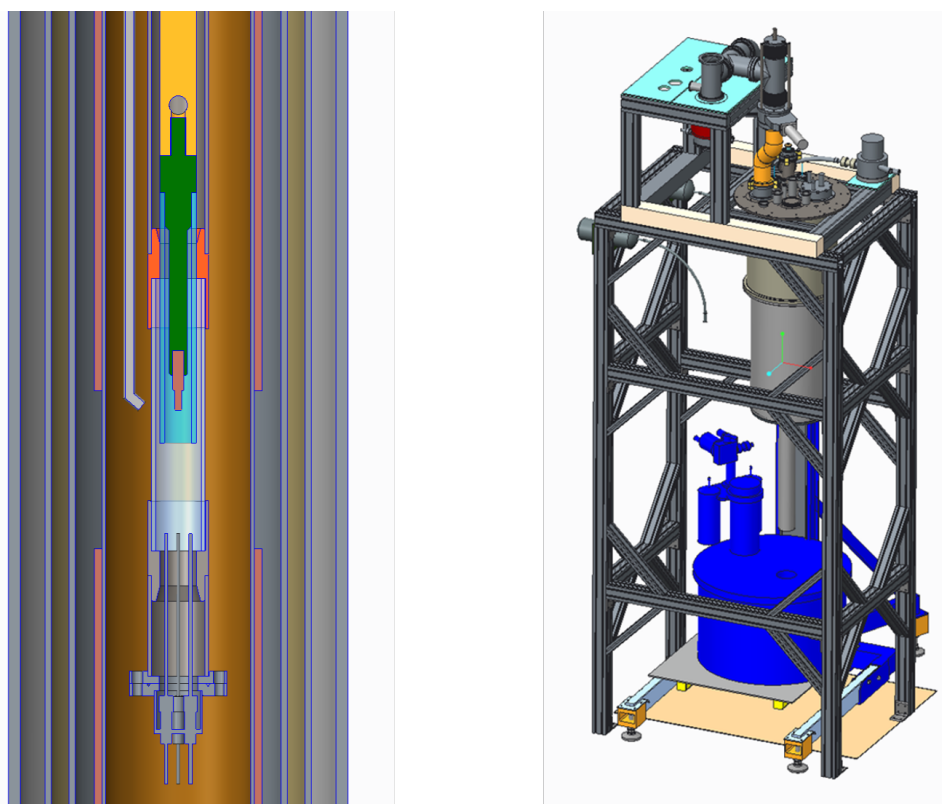

Figure 3: Left, a cross sectional view of the sample space. The sample space consists of a quartz tube, thermally connected to the mixing chamber of the refrigerator, and filled with a small amount of helium to act as an exchange gas. The sample itself would be at the bottom of the copper post in center. The microwaves are broadcast though the quartz tube into the sample space. Right, a model of the target, as it will be used on the beamline without the IMAGINE instrument. In this case, the refrigerator remains stationary and centered with the crystal on the beamline, and the magnet is moved up and down to repolarize the target. In order to interface the target with the IMAGINE detector, a more complicated elevator system will have to be built above the beamline

\section{Sample Material}

\subsection{Protein Crystals}

This project is envisioned as a proof of concept. As such the first proteins that will be looked at will be ones that are relatively well known. Most of the early work will be done with lysozyme crystals, which are relatively easy to produce in large quantities, and with crystals of a large size.

\subsection{Paramagnetic Centers}

Paramagnetic centers will be added to the crystals through the addition of a chemical dopant, mostly TEMPO. The simplest method will be to soak the crystals in a solution containing the TEMPO as well as a cryoprotectant such as glycerol, and freeze them. Additionally, there are a number of TEMPO based spin labels which are commercially available. These can be covalently bonded to sites on the protein. These methods will both be tested. Early tests conducted at the 
University of Virginia have shown promising polarizations for xylose isomerase crystals soaked in a TEMPO solution. To support a future user program, we will conduct EPR studies of samples, especially those that show a high degree of polarization. Representative spectra can be used by users to help evaluate how effective their samples have been prepared without having to waste time trying to polarize poorly doped samples.

\section{Outlook and Schedule}

This target has a very aggressive schedule, made so in part by the need to match with the schedule of the HFIR reactor, which runs six cycles a year. At this stage, the procurement of the polarizing magnet and dilution refrigerator are underway, the design of nearly all of the components is complete, and the first components are being fabricated. The target should be assembled in the spring of 2016. This will allow a few months of commissioning before the target takes beam at the CG-4D beamline during fall of 2016 .

\section{Acknowledgements}

Research sponsored by the Laboratory Directed Research and Development Program of Oak Ridge National Laboratory, managed by UT-Battelle, LLC, for the U. S. Department of Energy. The construction and installation of the IMAGINE beam line was partly supported by NSF grant CHE-0922719.

\section{References}

[1] Stuhrmann, H. B., et al. "Dynamic nuclear polarisation of biological matter." European Biophysics Journal 14.1 (1986): 1-6.

[2] Zhao, J., and H. B. Stuhrmann. "The in situ structure of the L3 and L4 proteins of the large subunit of E. coli ribosomes as determined by nuclear spin contrast variation." Le Journal de Physique IV 3.C8 (1993): C8-233.

[3] D.G. Crabb and W. Meyer, Annu. Rev. Nucl. Part. Sci. 47 (1997) 67.

[4] Meilleur, Flora, et al. "The IMAGINE instrument: first neutron protein structure and new capabilities for neutron macromolecular crystallography." Acta Crystallographica Section D: Biological Crystallography 69.10 (2013): 2157-2160.

[5] C.D. Keith, et al., Nuclear Instruments and Methods in Physics Research Section A 684 (2012) 27.

[6] G.R. Court, D.W. Gifford, P. Harrison, W.G. Heyes, and M.A. Houlden, Nuclear Instruments and Methods in Physics Research Section A 324 (1993) 433. 